\title{
Seismic activity on the territory of Slovakia in 2020
}

\author{
Róbert KYSEL ${ }^{1,2, *}$ (D) Andrej CIPCIAR $^{1,2}$ (D), Martin ŠUGÁR ${ }^{1,3}$ (D), \\ Kristián CSICSAY ${ }^{1}$ (D) Lucia FOJTÍKOVÁ ${ }^{1}$ iD, Peter PAŽÁK ${ }^{1}$ \\ ${ }^{1}$ Earth Science Institute of the Slovak Academy of Sciences, \\ Dúbravská cesta 9, P. O. Box 106, 84005 Bratislava, Slovak Republic \\ ${ }^{2}$ Faculty of Mathematics, Physics and Informatics, Comenius University in Bratislava, \\ Mlynská dolina, 84248 Bratislava, Slovak Republic \\ ${ }^{3}$ Faculty of Natural Sciences, Comenius University in Bratislava, \\ Mlynská dolina, Ilkovičova 6, 84215 Bratislava, Slovak Republic
}

\begin{abstract}
The National Network of Seismic Stations of Slovakia (NNSS) consists of eight short period and six broadband permanent seismic stations and a data centre located at the Earth Science Institute of the Slovak Academy of Sciences (ESI SAS). The NNSS recorded and detected 11229 seismic events from all epicentral distances in 2020. Totally 96 earthquakes originated in the territory of Slovakia in 2020. This paper provides basic information on the configuration of the NNSS, routine data processing, seismic activity on the territory of Slovakia in 2020 as well as macroseismic observations collected in 2020 .
\end{abstract}

Key words: Slovakia, National Network of Seismic Stations, seismicity, macroseismic observations

\section{Introduction}

The seismic activity on the territory of Slovakia and adjacent areas has been reported on the daily basis by the so called Seismo Reports published on the web sites of the Department of Seismology, ESI SAS http://www. seismo logy.sk/Seismo_Reports/reports.html and in annual reports as a part of the project Partial monitoring system - Geological factors (Liščák et al., 2021, in preparation) which is solved with a contract between ESI SAS and State Geological Institute of Dionýz Štúr. The aim of this paper is to provide a quick overview of earthquakes which originated on the territory of Slovakia or were macroseismically felt on the territory of Slovakia in 2020 .

\footnotetext{
*corresponding author, e-mail: robert.kysel@savba.sk
} 


\section{Seismic stations operating in 2020}

The seismic monitoring of the Slovak territory is provided by the NNSS operated by the ESI SAS (ESI SAS, 2004; Csicsay et al., 2018), Local Seismic Network in Eastern Slovakia operated by the Faculty of Mathematics, Physics and Informatics of the Comenius University in Bratislava and local network of seismic stations around NPPs Jaslovské Bohunice and Mochovce operated by Progseis Ltd. company. The networks of seismic stations cooperate, and the exchange of data is on the regular basis. The positions of the seismic stations on the territory of Slovakia are shown in Fig. 1.

In 2020 the NNSS consisted of thirteen permanent seismic stations, from which eight are short period and six are broadband. Broadband stations are: Kolonické sedlo (KOLS), Modra (MODS), Skalnaté pleso (SPC), Vyhne (VYHS) and Bratislava - Železná studnička (ZST). Short period stations are: Hurbanovo (HRB), Izabela (IZAB), Iža (SRO1), Kečovo (KECS), Liptovská Anna (LANS), Moča (SRO2), Stebnícka Huta (STHS), Šrobárová (SRO). The HRB is the oldest NNSS seismic station that has been in operation since 1909 (Pajdušák, 1997). Broadband station Cervenica (CRVS) located in Slanské Hills has been out of operation since 2018.

A new broadband station SPC has been built in cooperation with Institute of Geophysics of the Czech Academy of Sciences (GPI CAS) and

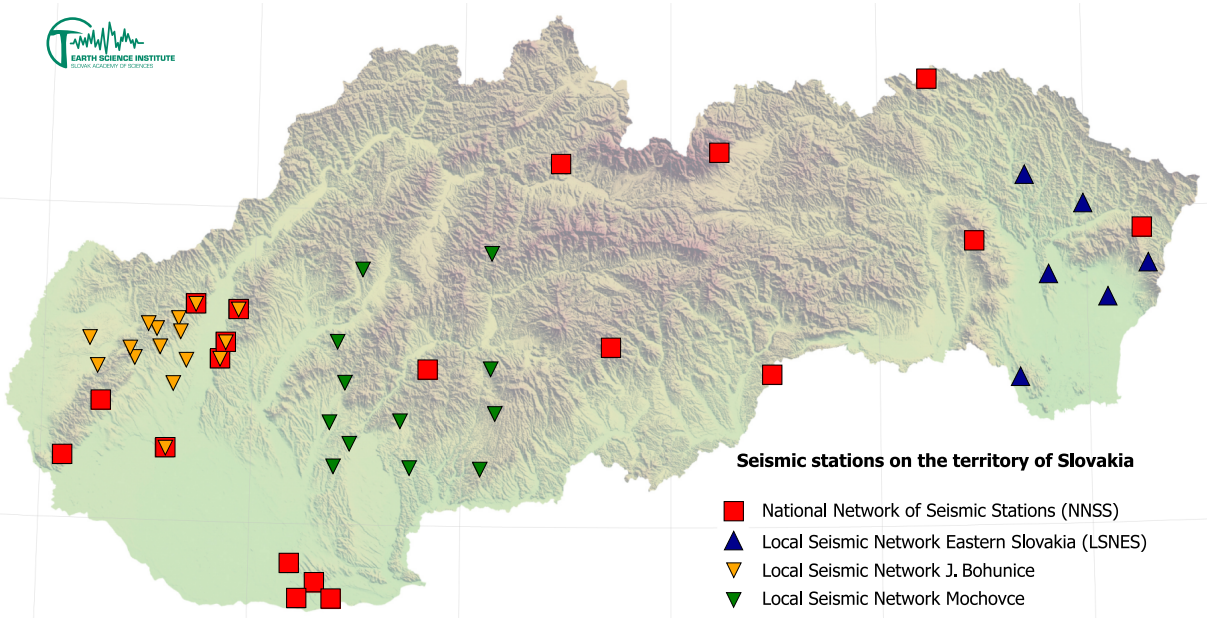

Fig. 1. Seismic stations operational on the territory of Slovakia in 2020. 
officially put into operation on July 21, 2020. The station is installed with a broadband sensor Guralp-CMG40T-30s and a digitizer Earth data PR624. The NNSS permanent seismic stations and their instrumentation are summarized in Table 1. More details can be found on the web page http:// www. seismology.sk/National Network.

Four additional short period seismic stations have been operated on the jointly bases of ESI SAS and other institutions. The seismic stations Banka (BAN), Podolie (POD) and Jalšové (JAL) located in the Little Carpathians have been operated in cooperation with Progseis Ltd. company and the Institute of Rock Structure and Mechanics of the Czech Academy of Sciences (IRSM CAS) (Fojtiková et al., 2015). A new seismic station was built in Považský Inovec Mts. nearby the Tematin castle ruins (TEMA) in cooperation with the IRSM CAS. The seismic station in Pusté Úlany (PULA) has been under reconstruction. These stations have been installed as temporary seismic stations.

Table 1. Equipment of seismic stations of the NNSS operating in 2020.

\begin{tabular}{|c|c|c|c|c|c|c|c|c|}
\hline Station & $\begin{array}{l}\text { ISC } \\
\text { code }\end{array}$ & $\begin{array}{l}\text { Lat. } \\
{\left[{ }^{\circ} \mathbf{N}\right]}\end{array}$ & $\begin{array}{l}\text { Long. } \\
{\left[{ }^{\circ} \mathbf{E}\right]}\end{array}$ & $\begin{array}{l}\text { Alt. } \\
{[\mathrm{m}]}\end{array}$ & Sensor & DAS & $\begin{array}{c}\text { Sampl. } \\
\text { freq. }\end{array}$ & $\begin{array}{c}\begin{array}{c}\text { Data } \\
\text { format }\end{array} \\
\end{array}$ \\
\hline $\begin{array}{c}\text { Bratislava } \\
\text { Žel. Studnička } \\
\end{array}$ & $\mathrm{ZST}$ & 48.196 & 17.102 & 250 & $3 \times \mathrm{SKD}$ & Wave32 & $100 / \mathrm{sec}$ & mSEED \\
\hline Vyhne & VYHS & 48.493 & 18.836 & 450 & STS-2 & Wave24 & $100 / \mathrm{sec}$ & mSEED \\
\hline Modra-Piesok & MODS & 48.373 & 17.277 & 520 & STS-2 & Wave32 & $100 / \mathrm{sec}$ & mSEED \\
\hline Hurbanovo & HRB & 47.873 & 18.192 & 115 & \begin{tabular}{|c}
$2 \times$ \\
Mainka \\
\end{tabular} & Analog & - & $\begin{array}{c}\text { smoked } \\
\text { paper }\end{array}$ \\
\hline Izabela & IZAB & 48.569 & 19.713 & 450 & $3 \times \mathrm{SM} 3$ & Wave24 & $100 / \mathrm{sec}$ & mSEED \\
\hline Iža & SRO1 & 47.7622 & 18.2328 & 111 & LE3D & $\mathrm{PCM}$ & $20 / \mathrm{sec}$ & mSEED \\
\hline Kečovo & KECS & 48.483 & 20.486 & 345 & LE3D & Wave24 & $100 / \mathrm{sec}$ & mSEED \\
\hline Kolonické sedlo & KOLS & 48.933 & 22.273 & 460 & \begin{tabular}{|l} 
Guralp- \\
6T-30s
\end{tabular} & Wave32 & $100 / \mathrm{sec}$ & mSEED \\
\hline Liptovská Anna & LANS & 49.151 & 19.468 & 710 & LE3D & SEMS & $100 / \mathrm{sec}$ & mSEED \\
\hline Moča & SRO2 & 47.763 & 18.394 & 109 & \begin{tabular}{|c|} 
Guralp- \\
40T-1s \\
\end{tabular} & Wave24 & $100 / \mathrm{sec}$ & mSEED \\
\hline Stebnícka Huta & STHS & 49.417 & 21.244 & 534 & LE3D & Wave24 & $100 / \mathrm{sec}$ & mSEED \\
\hline Šrobárová & $\mathrm{SRO}$ & 47.813 & 18.313 & 150 & \begin{tabular}{|c}
$3 \times$ \\
SKM-3 \\
\end{tabular} & Wave24 & $100 / \mathrm{sec}$ & mSEED \\
\hline Skalnaté Pleso & SPC & 49.189 & 20.234 & 1751 & \begin{tabular}{|l} 
Guralp- \\
40T-30s
\end{tabular} & $\begin{array}{l}\text { SeisComp } \\
\text { EarthData }\end{array}$ & $100 / \mathrm{sec}$ & mSEED \\
\hline
\end{tabular}




\section{Data processing}

Digital data from all NNSS stations (except the analog seismic station HRB) are transferred in real-time to the data centre at the ESI SAS either by the internet or satellite telemetry. Software package SeisComp3 (Weber et al., 2007) and SeedLink server are used for data acquisition and exchange.

Beside observations from the NNSS stations the data center at ESI SAS also use the data from the above-mentioned local networks of seismic stations in Slovakia and observations from networks of neighbouring countries: Austrian Seismic Network (ZAMG - Zentralanstalt für Meterologie und Geodynamik, 1987), Czech Regional Seismic Network (Institute of Geophysics, Academy of Sciences of the Czech Republic, 1973), Hungarian National Seismological Network (Kövesligethy Radó Seismological Observatory, 1992), Local seismological network for monitoring NPP Dukovany (Institute of Physics of the Earth Masaryk University, 2014), GEOFON Seismic Network (GEOFON Data Center, 1993), Polish Seismological Network. These stations form a so-called Regional Virtual Network of ESI SAS that consists of approximately 55 seismic stations.

Routine analysis of the digital recordings at the ESI SAS has been performed by the Unix package Seismic handler (Stammler, 1993). Interactive locations of seismic events within Seismic Handler are performed by external program LocSat. The collected digital observations are manually processed on the daily basis. The epicentre locations are based on the IASP91 traveltime curves. Local magnitudes have been determined from the maximum vertical trace amplitudes of Sg waves, using the pre-defined Seismic Handler formula for local events. Continuous raw seismic data from the NNSS are stored in a local archive and seismic data interpretations (together with information on equipment of stations) are stored in a web accessible database.

\section{Seismic activity in 2020}

The NNSS analyzed 11229 local, regional and teleseismic events in 2020. More than 35000 seismic phases were determined. Seismic events identified as quarry blasts were excluded from further processing and were not included in the event statistics. All events recorded by the NNSS and analyses at the data centre at ESI SAS has been reported in the so called Seismo Reports and published on the above-mentioned web sites of the Department 
of Seismology, ESI SAS.

Altogether 96 seismic events located by the NNSS originated in the territory of Slovakia in 2020 (Fig. 2). Known quarry blasts are not included in this number. 27 earthquakes reached local magnitude 1.0 or more and are listed in Table 2. The strongest earthquake occurred April 23 at 23:18 UTC in Vihorlat Mts. with local magnitude $M_{L} 3.3$.

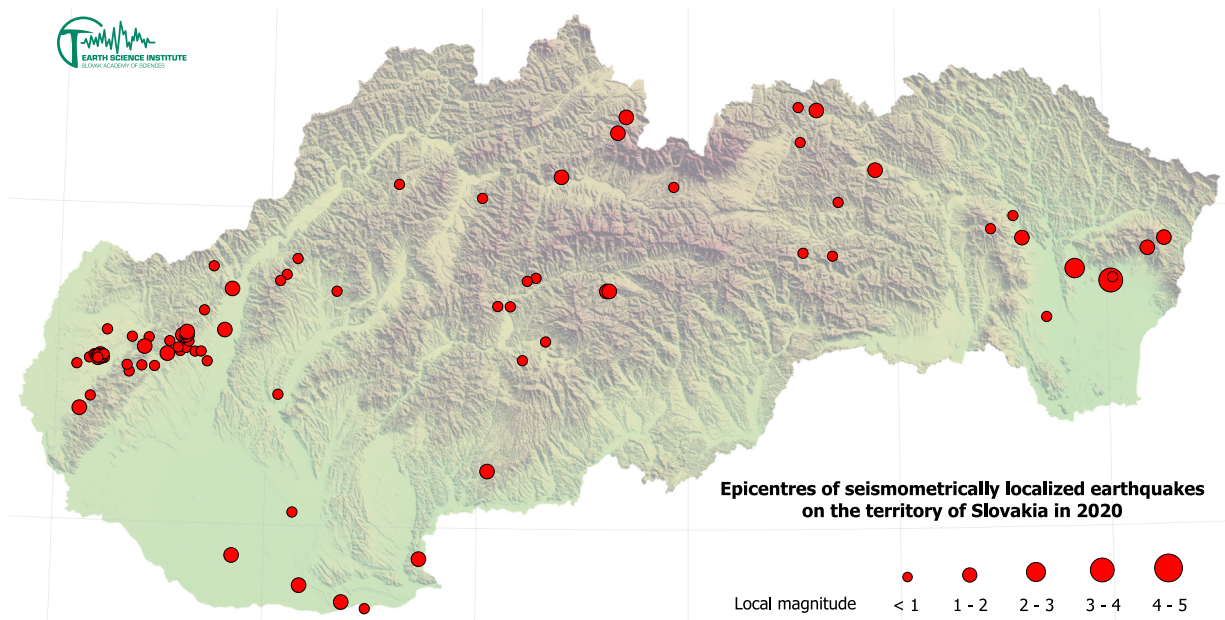

Fig. 2. Map of epicentres of local earthquakes originated on the territory of Slovakia in 2020. Diameters of the circles are proportional to local magnitudes.

The seismicity of the Slovak territory is dominated by the Little Carpathians and Komárno seismic source zones (Hók et al., 2016). The strongest earthquake in the Little Carpathians source zone was detected on the December 24 at 15:34 UTC with local magnitude $M_{L}$ 1.6. The strongest earthquake in the Komárno source zone was detected on the August 15 at 6:22 UTC with local magnitude $M_{L}$ 1.4. Although the low level of seismic activity from the last decades remained unchanged also in 2020, these two source zones remain of the primary interest for the monitoring of seismic activity within the territory of Slovakia.

\section{Macroseismic observations}

Six earthquakes were macroseismically felt on the territory of Slovakia in 2020 (Table 3), four of them with the epicentre on the territory of Slovakia 
Table 2. List of earthquakes originated on the territory of Slovakia in 2020 with $M_{L} \geq 1.0$.

\begin{tabular}{|c|c|c|c|c|c|c|c|}
\hline $\begin{array}{c}\text { Date } \\
{[\text { YYYY-MM-DD] }}\end{array}$ & $\begin{array}{c}\text { Origin Time } \\
\text { (UTC) } \\
\text { [HH:MM:SS] }\end{array}$ & $\begin{array}{l}\text { Lat. } \\
{\left[{ }^{\circ} \mathbf{N}\right]}\end{array}$ & $\begin{array}{l}\text { Lon. } \\
{\left[{ }^{\circ} \mathbf{E}\right]}\end{array}$ & $\begin{array}{l}\text { Depth } \\
{[\mathrm{km}]}\end{array}$ & $\begin{array}{c}M_{L} \\
{[\mathrm{NNSS}]}\end{array}$ & {$\left[\begin{array}{c}\boldsymbol{I}_{0} \\
{\left[{ }^{\circ} \mathbf{E M S}\right]}\end{array}\right.$} & Region \\
\hline 2020-01-25 & 09:20:20.57 & 49.11 & 19.39 & 0 & 1.0 & & Liptov region \\
\hline 2020-02-08 & $20: 42: 26.23$ & 48.51 & 17.19 & 0 & 1.7 & 3 & Záhorie region \\
\hline 2020-02-13 & $16: 28: 14.40$ & 48.52 & 17.20 & 2 & 1.0 & & Záhorie region \\
\hline 2020-03-01 & $10: 57: 54.91$ & 48.35 & 17.11 & 0 & 1.0 & & $\begin{array}{c}\text { Little } \\
\text { Carpathians }\end{array}$ \\
\hline 2020-04-17 & 15:54:08.47 & 48.91 & 21.60 & 1 & 1.0 & & Slanské Hills \\
\hline $2020-04-23$ & $23: 18: 27.21$ & 48.77 & 22.02 & 16 & 3.3 & $5-6$ & Vihorlat Mts. \\
\hline 2020-04-30 & 03:59:05.65 & 48.81 & 21.85 & 8 & 2.3 & 4 & Vihorlat Mts. \\
\hline 2020-06-11 & 22:19:58.39 & 48.18 & 19.05 & 0 & 1.0 & & Krupina Plain \\
\hline 2020-06-22 & 06:43:37.04 & 48.87 & 22.20 & 0 & 1.2 & & Vihorlat Mts. \\
\hline $2020-07-16$ & $17: 45: 46.03$ & 48.59 & 17.59 & 3 & 1.3 & & $\begin{array}{c}\text { Little } \\
\text { Carpathians }\end{array}$ \\
\hline 2020-07-22 & $10: 57: 28.47$ & 49.32 & 20.62 & 0 & 1.3 & & Pieniny Mts. \\
\hline 2020-08-12 & 18:07:08.09 & 48.90 & 22.28 & 6 & 1.9 & & Vihorlat Mts. \\
\hline 2020-08-15 & $06: 22: 37.76$ & 47.81 & 18.17 & 0 & 1.4 & & $\begin{array}{l}\text { Šamorín - } \\
\text { Komárno - } \\
\text { Štúrovo }\end{array}$ \\
\hline $2020-08-20$ & 03:53:22.61 & 48.53 & 17.52 & 2 & 1.2 & & $\begin{array}{c}\text { Little } \\
\text { Carpathians } \\
\end{array}$ \\
\hline 2020-08-31 & $07: 50: 25.86$ & 48.75 & 19.61 & 0 & 1.6 & 3 & Vepor Mts. \\
\hline 2020-09-01 & 03:59:12.83 & 48.75 & 19.62 & 0 & 1.2 & & Vepor Mts. \\
\hline 2020-09-13 & 00:03:52.72 & 48.59 & 17.61 & 3 & 1.1 & & $\begin{array}{c}\text { Little } \\
\text { Carpathians }\end{array}$ \\
\hline 2020-09-15 & 08:58:39.66 & 49.13 & 20.90 & 10 & 1.6 & & Levoča Mts. \\
\hline 2020-09-20 & $07: 37: 25.65$ & 47.90 & 17.85 & 12 & 1.3 & & $\begin{array}{c}\text { Šamorín - } \\
\text { Komárno - } \\
\text { Štúrovo }\end{array}$ \\
\hline 2020-09-21 & 10:17:06.78 & 49.25 & 19.66 & 0 & 1.1 & & $\begin{array}{c}\text { Western } \\
\text { Tatras }\end{array}$ \\
\hline 2020-09-25 & 19:21:08.48 & 48.60 & 17.61 & 3 & 1.4 & & $\begin{array}{c}\text { Little } \\
\text { Carpathians }\end{array}$ \\
\hline 2020-10-09 & $10: 24: 17.22$ & 49.30 & 19.70 & 0 & 1.4 & & $\begin{array}{l}\text { Western } \\
\text { Tatras }\end{array}$ \\
\hline
\end{tabular}


Table 2. Continued from the previous page.

\begin{tabular}{|c|c|c|c|c|c|c|c|}
\hline $\begin{array}{c}\text { Date } \\
{[\text { YYYY-MM-DD] }}\end{array}$ & $\begin{array}{c}\text { Origin Time } \\
\text { (UTC) } \\
\text { [HH:MM:SS] }\end{array}$ & $\begin{array}{l}\text { Lat. } \\
{\left[{ }^{\circ} \mathbf{N}\right]}\end{array}$ & $\begin{array}{l}\text { Lon. } \\
{\left[{ }^{\circ} \mathbf{E}\right]}\end{array}$ & $\begin{array}{c}\text { Depth } \\
{[\mathrm{km}]}\end{array}$ & $\begin{array}{c}M_{L} \\
{[\mathrm{NNSS}]}\end{array}$ & {$\left[\begin{array}{c}\boldsymbol{I}_{0} \\
{\left[{ }^{\circ} \mathbf{E M S}\right]}\end{array}\right.$} & Region \\
\hline $2020-10-29$ & 09:39:48.38 & 48.61 & 17.79 & 0 & 1.2 & & $\begin{array}{c}\text { Trnava } \\
\text { Highlands }\end{array}$ \\
\hline $2020-11-30$ & 08:42:33.49 & 47.90 & 18.73 & 5 & 1.4 & & $\begin{array}{l}\text { Šamorín - } \\
\text { Komárno - } \\
\text { Štúrovo }\end{array}$ \\
\hline $2020-11-30$ & $19: 18: 39.25$ & 47.76 & 18.37 & 7 & 1.3 & & $\begin{array}{l}\text { Šamorín - } \\
\text { Komárno - } \\
\text { Štúrovo }\end{array}$ \\
\hline $2020-12-24$ & $15: 34: 02.40$ & 48.74 & 17.82 & 5 & 1.6 & & $\begin{array}{c}\text { Little } \\
\text { Carpathians }\end{array}$ \\
\hline $2020-12-25$ & $08: 43: 36.08$ & 48.55 & 17.41 & 0 & 1.3 & & $\begin{array}{c}\text { Little } \\
\text { Carpathians }\end{array}$ \\
\hline
\end{tabular}

Table 3. List of macroseismically observed earthquakes on the territory of Slovakia in 2020 .

\begin{tabular}{|c|c|c|c|c|c|c|c|}
\hline $\begin{array}{c}\text { Date } \\
\text { [YYYY-MM-DD] }]\end{array}$ & $\begin{array}{c}\text { Origin Time } \\
\text { (UTC) } \\
{[\mathbf{H H : M M : S S}]}\end{array}$ & $\begin{array}{c}\text { Lat. } \\
{\left[{ }^{\circ} \mathbf{N}\right]}\end{array}$ & $\begin{array}{c}\text { Lon. } \\
{\left[{ }^{\circ} \mathbf{E}\right]}\end{array}$ & $\begin{array}{c}\text { Depth } \\
{[\mathbf{k m}]}\end{array}$ & $\begin{array}{c}\boldsymbol{M}_{\boldsymbol{L}} \\
{[\mathbf{N N S S}]}\end{array}$ & $\begin{array}{c}\boldsymbol{I}_{0} \\
{\left[{ }^{\circ} \mathbf{E M S}\right]}\end{array}$ & Region \\
\hline $2020-02-08$ & $20: 42: 26.2$ & 48.51 & 17.19 & 0 & 1.7 & 3 & Záhorie region \\
\hline $2020-03-22$ & $05: 24: 02.8$ & 45.87 & 16.02 & 10 & 5.2 & $3^{*}$ & Croatia \\
\hline $2020-04-23$ & $23: 18: 27.2$ & 48.77 & 22.02 & 16 & 3.3 & $5-6$ & Vihorlat Mts. \\
\hline $2020-04-30$ & $03: 59: 05.7$ & 48.81 & 21.85 & 8 & 2.3 & 4 & Vihorlat Mts. \\
\hline $2020-08-31$ & $07: 50: 25.9$ & 48.75 & 19.61 & 0 & 1.6 & 3 & Vepor Mts. \\
\hline $2020-12-29$ & $11: 19: 54.6$ & 45.46 & 16.31 & 10 & 6.2 & $4^{*}$ & Croatia \\
\hline
\end{tabular}

* highest intensity on the territory of Slovakia

(Fig. 3) and two of them on the territory of Croatia. All of these six earthquakes were also seismometrically observed and processed. Intensities were estimated by the European Macroseismic Scale 1998 (EMS-98) introduced by Grünthal (1998).

The earthquake on February 8 at 20:42 UTC with epicentre in Záhorie region between municipalities Plavecké Podhradie and Studienka and local magnitude 1.7 was macroseismically felt on 2 locations (Table 4). 5 macroseimic questionnaires were filled. The epicentral intensity was determined at $3^{\circ}$ EMS-98. 


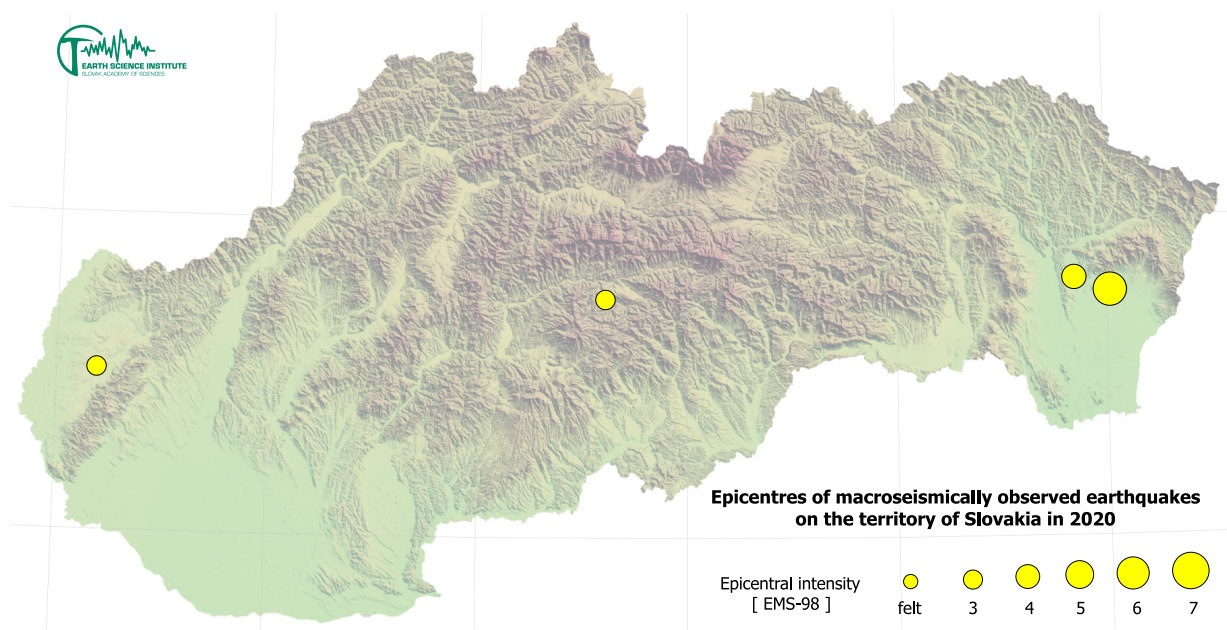

Fig. 3. Map of epicentres of macroseimically observed earthquakes on the territory of Slovakia in 2020. Diameters of the circles are proportional to epicentral intensity.

The earthquake on March 22 at 5:24 UTC with epicentre near Zagreb, Croatia and local magnitude 5.2 was macroseismically felt on 6 locations within the territory of Slovakia (Table 5). 24 macroseismic questionnaires were filled. People reported weak trembling on higher floors in municipalities Bratislava and Nové Zámky. The maximum intensity on the territory of Slovakia was determined at $3^{\circ}$ EMS-98.

The earthquake on April 23 at 23:18 UTC with epicentre in Vihorlat Mts. located between municipalities Malé Zálužice and Lúčky and local magnitude 3.3 was macroseismically felt on 56 locations (Table 6). 544 macroseimic questionnaires were filled. People reported moderate shaking, cracks in walls, felt small pieces of plaster and partly damaged chimneys. The epicentral intensity was determined at $5-6^{\circ}$ EMS-98. Due to the Covid-19 pandemics lockdown and travelling restrictions an on-site inspection of reported damages was not possible.

The earthquake on April 30 at 3:59 UTC with epicentre in Vihorlat Mts. located between municipalities Nacina Ves and Lesné and local magnitude 2.3 was macroseismically felt on 18 locations (Table 7 ). 70 macroseismic questionnaires were filled. The event was described as a trembling. Some people reported acoustic effects - rumble and shaking of light furniture. The epicentral intensity was determined at $4^{\circ}$ EMS-98. 
Table 4. Macroseismic observations for February 8, 2020 earthquake, 20:42 UTC.

\begin{tabular}{|l|c|c|c|c|}
\hline Locality & $\begin{array}{c}\text { Lat. } \\
{\left[{ }^{\circ} \mathbf{N}\right]}\end{array}$ & $\begin{array}{c}\text { Lon. } \\
{\left[{ }^{\circ} \mathbf{E}\right]}\end{array}$ & $\begin{array}{c}\text { No. of } \\
\text { questionnaires }\end{array}$ & $\begin{array}{c}\boldsymbol{I} \\
{\left[{ }^{\circ} \text { EMS-98 }\right]}\end{array}$ \\
\hline Plavecký Mikuláš & 48.519 & 17.306 & 4 & 3 \\
\hline Plavecký Peter & 48.536 & 17.327 & 1 & 3 \\
\hline
\end{tabular}

Table 5. Macroseismic observations for March 22, 2020 earthquake, 5:24 UTC.

\begin{tabular}{|l|c|c|c|c|}
\hline Locality & $\begin{array}{c}\text { Lat. } \\
{\left[{ }^{\circ} \mathbf{N}\right]}\end{array}$ & $\begin{array}{c}\text { Lon. } \\
{\left[{ }^{\circ} \mathbf{E}\right]}\end{array}$ & $\begin{array}{c}\text { No. of } \\
\text { questionnaires }\end{array}$ & $\begin{array}{c}\text { I } \\
{\left[{ }^{\circ} \text { EMS-98 }\right]}\end{array}$ \\
\hline Nové Zámky & 47.993 & 18.170 & 12 & 3 \\
\hline Bratislava & 48.163 & 17.126 & 7 & 3 \\
\hline Sered' & 48.289 & 17.729 & 2 & 3 \\
\hline Komárno & 47.766 & 18.118 & 1 & 3 \\
\hline Nové Mesto nad Váhom & 48.758 & 17.829 & 1 & 3 \\
\hline Pezinok & 48.298 & 17.270 & 1 & 3 \\
\hline
\end{tabular}

Table 6. Macroseismic observations for April 23, 2020 earthquake, 23:18 UTC.

\begin{tabular}{|l|c|c|c|c|}
\hline Locality & $\begin{array}{c}\text { Lat. } \\
{\left[{ }^{\circ} \mathbf{N}\right]}\end{array}$ & $\begin{array}{c}\text { Lon. } \\
{\left[{ }^{\circ} \mathbf{E}\right]}\end{array}$ & $\begin{array}{c}\text { No. of } \\
\text { questionnaires }\end{array}$ & $\begin{array}{c}\text { I } \\
{\left[{ }^{\circ} \text { EMS-98 }\right]}\end{array}$ \\
\hline Michalovce & 48.753 & 21.915 & 379 & $5-6$ \\
\hline Zalužice & 48.757 & 21.991 & 29 & $5-6$ \\
\hline Petrovce nad Laborcom & 48.794 & 21.868 & 7 & $5-6$ \\
\hline Vinné & 48.814 & 21.977 & 14 & 5 \\
\hline Hažín & 48.752 & 22.021 & 10 & 5 \\
\hline Kaluža & 48.806 & 22.006 & 5 & $5 ?$ \\
\hline Betlenovce & 48.729 & 21.903 & 2 & $5 ?$ \\
\hline Močarany & 48.739 & 21.872 & 2 & $5 ?$ \\
\hline Šamudovce & 48.708 & 21.888 & 2 & 5 \\
\hline Hatalov & 48.659 & 21.889 & 1 & 5 \\
\hline Sobrance & 48.756 & 22.189 & 1 & 5 \\
\hline Vrbnica & 48.685 & 21.885 & 1 & $5 ?$ \\
\hline Trnava pri Laborci & 48.820 & 21.933 & 6 & $4-5$ \\
\hline Vel'ké Zalužice & 48.757 & 21.978 & 6 & $4-5$ \\
\hline Nacina Ves & 48.823 & 21.842 & 5 & $4-5$ \\
\hline Krásnovce & 48.722 & 21.901 & 3 & $4-5$ \\
\hline
\end{tabular}


Table 6. Continued from the previous page.

\begin{tabular}{|c|c|c|c|c|}
\hline Locality & $\begin{array}{l}\text { Lat. } \\
{\left[{ }^{\circ} \mathbf{N}\right]}\end{array}$ & $\begin{array}{l}\text { Lon. } \\
{\left[{ }^{\circ} \mathbf{E}\right]}\end{array}$ & $\begin{array}{c}\text { No. of } \\
\text { questionnaires }\end{array}$ & $\begin{array}{c}I \\
{\left[{ }^{\circ} \text { EMS-98] }\right.}\end{array}$ \\
\hline Lastomír & 48.706 & 21.929 & 3 & $4-5$ \\
\hline Čečehov & 48.723 & 21.994 & 2 & $4-5$ \\
\hline Lúčky & 48.767 & 22.045 & 2 & $4-5$ \\
\hline Sliepkovce & 48.668 & 21.942 & 2 & $4-5$ \\
\hline Med'ov & 48.737 & 21.936 & 1 & $4-5$ \\
\hline Milovaná & 48.763 & 21.866 & 2 & $4 ?$ \\
\hline Vel'ké Revištia & 48.750 & 22.109 & 2 & 4 \\
\hline Pichne & 49.039 & 22.123 & 1 & 4 \\
\hline Suché & 48.765 & 21.837 & 1 & 4 \\
\hline Rakovec nad Ondavou & 48.771 & 21.786 & 3 & $3-4$ \\
\hline Pozdišovce & 48.728 & 21.857 & 8 & 3 \\
\hline Humenné & 48.933 & 21.909 & 5 & 3 \\
\hline Strážske & 48.874 & 21.833 & 4 & 3 \\
\hline Moravany & 48.729 & 21.790 & 3 & 3 \\
\hline Trebišov & 48.626 & 21.716 & 2 & 3 \\
\hline Beša & 48.536 & 21.952 & 1 & 3 \\
\hline Brekov & 48.902 & 21.838 & 1 & 3 \\
\hline Komárovce & 48.654 & 21.650 & 1 & 3 \\
\hline Martin & 49.087 & 18.915 & 1 & 3 \\
\hline Lučkovce & 48.720 & 21.785 & 1 & 3 \\
\hline Poša & 48.839 & 21.756 & 1 & 3 \\
\hline Vranov nad Toplou & 48.882 & 21.689 & 2 & 3 \\
\hline Vyšný Žipov & 48.987 & 21.590 & 1 & 3 \\
\hline Závadka & 48.928 & 21.842 & 1 & 3 \\
\hline Žbince & 48.671 & 21.887 & 1 & 3 \\
\hline Zemplínska Šírava & - & - & 3 & felt \\
\hline Klokočov & 48.813 & 22.032 & 2 & felt \\
\hline Topol'any & 48.785 & 21.867 & 2 & felt \\
\hline Babin Potok & 49.117 & 21.223 & 1 & felt \\
\hline Bajany & 48.603 & 22.111 & 1 & felt \\
\hline Budkovce & 48.629 & 21.944 & 1 & felt \\
\hline
\end{tabular}


Table 6. Continued from the previous page.

\begin{tabular}{|l|c|c|c|c|}
\hline Locality & $\begin{array}{c}\text { Lat. } \\
{\left[{ }^{\circ} \mathbf{N}\right]}\end{array}$ & $\begin{array}{c}\text { Lon. } \\
{\left[{ }^{\circ} \mathbf{E}\right]}\end{array}$ & $\begin{array}{c}\text { No. of } \\
\text { questionnaires }\end{array}$ & $\begin{array}{c}\boldsymbol{I} \\
{\left[{ }^{\circ} \text { EMS-98 }\right]}\end{array}$ \\
\hline Čerhov & 48.461 & 21.644 & 1 & felt \\
\hline Dlhé Klčovo & 48.812 & 21.739 & 1 & felt \\
\hline Jasenov & 48.797 & 22.173 & 1 & felt \\
\hline Lesné & 48.799 & 21.817 & 1 & felt \\
\hline Nižný Hrušov & 48.810 & 21.776 & 1 & felt \\
\hline Porúbka & 49.200 & 21.430 & 1 & felt \\
\hline Senné & 48.665 & 22.030 & 1 & felt \\
\hline Vybúchanec & 48.821 & 21.816 & 1 & felt \\
\hline Zbudza & 48.816 & 21.895 & 1 & felt \\
\hline
\end{tabular}

Table 7. Macroseismic observations for April 30, 2020 earthquake, 3:59 UTC.

\begin{tabular}{|l|c|c|c|c|}
\hline Locality & $\begin{array}{c}\text { Lat. } \\
{\left[{ }^{\circ} \mathbf{N}\right]}\end{array}$ & $\begin{array}{c}\text { Lon. } \\
{\left[{ }^{\circ} \mathbf{E}\right]}\end{array}$ & $\begin{array}{c}\text { No. of } \\
\text { questionnaires }\end{array}$ & $\begin{array}{c}\text { I } \\
{\left[{ }^{\circ} \text { EMS-98 }\right]}\end{array}$ \\
\hline Pusté Čemerné & 48.843 & 21.819 & 7 & 4 \\
\hline Oreské & 48.824 & 19.604 & 3 & 4 \\
\hline Trnava pri Laborci & 48.820 & 21.933 & 5 & $3-4$ \\
\hline Michalovce & 48.753 & 21.915 & 9 & 3 \\
\hline Petrovce nad Laborcom & 48.794 & 21.868 & 9 & 3 \\
\hline Vinné & 48.814 & 21.977 & 9 & 3 \\
\hline Nacina Ves & 48.823 & 21.842 & 4 & 3 \\
\hline Staré & 48.412 & 21.903 & 4 & 3 \\
\hline Nižný Hrušov & 48.810 & 21.776 & 3 & 3 \\
\hline Poša & 48.839 & 21.756 & 3 & 3 \\
\hline Strážske & 48.874 & 21.833 & 3 & 3 \\
\hline Zbudza & 48.816 & 21.895 & 2 & 3 \\
\hline Zemplínska Š́rava & 48.787 & 22.012 & 1 & felt \\
\hline Dlhé Klčovo & 48.812 & 21.739 & 2 & felt \\
\hline Lesné & 48.799 & 21.817 & 2 & felt \\
\hline Nižný Hrabovec & 48.853 & 21.754 & 2 & felt \\
\hline Vola & 48.840 & 21.851 & 1 & felt \\
\hline Vybúchanec & 48.821 & 21.816 & 1 & \\
\hline
\end{tabular}


The earthquake on August 31 at 7:50 UTC with epicentre in Vepor Mts. located near municipality Čierny Balog and local magnitude 1.6 was macroseismically felt on 1 location (Table 8). One macroseimic questionnaire was filled. The epicentral intensity was determined at $3^{\circ}$ EMS-98.

The earthquake on December 29 at 11:19 UTC with epicentre near Petrinja, Croatia and local magnitude 6.2 was macroseismically felt on 85 locations within the territory of Slovakia (Table 9). 682 macroseismic questionnaires were filled. People reported weak trembling on higher floors in municipalities Bratislava and Nitra. The maximum intensity on the territory of Slovakia was determined at $4^{\circ}$ EMS-98.

Table 8. Macroseismic observations for August 31, 2020 earthquake, 7:50 UTC.

\begin{tabular}{|l|c|c|c|c|}
\hline Locality & $\begin{array}{c}\text { Lat. } \\
{\left[{ }^{\circ} \mathbf{N}\right]}\end{array}$ & $\begin{array}{c}\text { Lon. } \\
{\left[{ }^{\circ} \mathbf{E}\right]}\end{array}$ & $\begin{array}{c}\text { No. of } \\
\text { questionnaires }\end{array}$ & $\begin{array}{c}\boldsymbol{I} \\
{\left[{ }^{\circ} \mathbf{E M S}-98\right]}\end{array}$ \\
\hline Brezno & 48.813 & 19.660 & 1 & 3 \\
\hline
\end{tabular}

Table 9. Macroseismic observations for December 29, 2020 earthquake, 11:19 UTC.

\begin{tabular}{|l|c|c|c|c|}
\hline Locality & $\begin{array}{c}\text { Lat. } \\
{\left[{ }^{\circ} \mathbf{N}\right]}\end{array}$ & $\begin{array}{c}\text { Lon. } \\
{\left[{ }^{\circ} \mathbf{E}\right]}\end{array}$ & $\begin{array}{c}\text { No. of } \\
\text { questionnaires }\end{array}$ & $\begin{array}{c}\text { I } \\
{\left[{ }^{\circ} \text { EMS-98 }\right]}\end{array}$ \\
\hline Lehnice & 48.048 & 17.458 & 1 & 4 \\
\hline Bratislava 5 & 48.068 & 17.119 & 184 & 3 \\
\hline Bratislava 2 & 48.139 & 17.192 & 119 & 3 \\
\hline Bratislava 4 & 48.204 & 17.028 & 68 & 3 \\
\hline Bratislava 1 & 48.147 & 17.102 & 55 & 3 \\
\hline Nitra & 48.314 & 18.092 & 34 & 3 \\
\hline Bratislava 3 & 48.207 & 17.147 & 20 & 3 \\
\hline Trnava & 48.378 & 17.587 & 18 & 3 \\
\hline Nové Zámky & 47.993 & 18.170 & 15 & 3 \\
\hline Sered' & 48.289 & 17.729 & 15 & 3 \\
\hline Komárno & 47.766 & 18.118 & 11 & 3 \\
\hline Levice & 48.217 & 18.610 & 9 & 3 \\
\hline Pezinok & 48.298 & 17.270 & 9 & 3 \\
\hline Šala & 48.151 & 17.888 & 8 & 3 \\
\hline Banská Bystrica & 48.733 & 19.143 & 7 & 6 \\
\hline Senec & 48.243 & 17.380 & & \\
\hline
\end{tabular}


Table 9. Continued from the previous page.

\begin{tabular}{|c|c|c|c|c|}
\hline Locality & $\begin{array}{l}\text { Lat. } \\
{\left[{ }^{\circ} \mathbf{N}\right]}\end{array}$ & $\begin{array}{l}\text { Lon. } \\
{\left[{ }^{\circ} \mathbf{E}\right]}\end{array}$ & $\begin{array}{c}\text { No. of } \\
\text { questionnaires }\end{array}$ & $\begin{array}{c}I \\
{\left[{ }^{\circ} \text { EMS-98 }\right]}\end{array}$ \\
\hline Nové Mesto nad Váhom & 48.758 & 17.829 & 5 & 3 \\
\hline Trenčín & 48.890 & 18.043 & 5 & 3 \\
\hline Dunajská Streda & 47.996 & 17.617 & 4 & 3 \\
\hline Bernolákovo & 48.200 & 17.300 & 3 & 3 \\
\hline Galanta & 48.198 & 17.734 & 3 & 3 \\
\hline Hlohovec & 48.431 & 17.798 & 3 & 3 \\
\hline Martin & 49.087 & 18.915 & 3 & 3 \\
\hline Michalovce & 48.753 & 21.915 & 3 & 3 \\
\hline Stupava & 48.269 & 17.023 & 3 & 3 \\
\hline Topol'čany & 48.561 & 18.174 & 3 & 3 \\
\hline Dunajská Lužná & 48.083 & 17.265 & 2 & 3 \\
\hline Lučenec & 48.332 & 19.664 & 2 & 3 \\
\hline Modra & 48.347 & 17.313 & 2 & 3 \\
\hline Partizánske & 48.641 & 18.589 & 2 & 3 \\
\hline Pieštany & 48.594 & 17.824 & 2 & 3 \\
\hline Rovinka & 48.098 & 17.237 & 2 & 3 \\
\hline Slovenský Grob & 48.256 & 17.279 & 2 & 3 \\
\hline Šamorín & 48.025 & 17.339 & 2 & 3 \\
\hline Balog nad Iplom & 48.078 & 19.133 & 1 & 3 \\
\hline Bobrovec & 49.122 & 19.616 & 1 & 3 \\
\hline Bohdanovce nad Trnavou & 48.424 & 17.541 & 1 & 3 \\
\hline Borský Mikuláš & 48.622 & 17.205 & 1 & 3 \\
\hline Báb & 48.307 & 17.880 & 1 & 3 \\
\hline Čachtice & 48.713 & 17.789 & 1 & 3 \\
\hline Dlhá nad Váhom & 48.171 & 17.863 & 1 & 3 \\
\hline Dudince & 48.169 & 18.885 & 1 & 3 \\
\hline Holíč & 48.810 & 17.161 & 1 & 3 \\
\hline Horné Saliby & 48.111 & 17.761 & 1 & 3 \\
\hline Horné Trhovište & 48.468 & 17.869 & 1 & 3 \\
\hline Hronovce & 48.003 & 18.645 & 1 & 3 \\
\hline Ivančiná & 48.911 & 18.814 & 1 & 3 \\
\hline
\end{tabular}


Table 9. Continued from the previous page.

\begin{tabular}{|c|c|c|c|c|}
\hline Locality & $\begin{array}{l}\text { Lat. } \\
{\left[{ }^{\circ} \mathbf{N}\right]}\end{array}$ & $\begin{array}{l}\text { Lon. } \\
{\left[{ }^{\circ} \mathbf{E}\right]}\end{array}$ & $\begin{array}{c}\text { No. of } \\
\text { questionnaires }\end{array}$ & $\begin{array}{c}I \\
{\left[{ }^{\circ} \text { EMS-98] }\right.}\end{array}$ \\
\hline Kmet'ovce & 48.262 & 18.708 & 1 & 3 \\
\hline Kovarce & 48.498 & 18.157 & 1 & 3 \\
\hline Košice & 48.711 & 21.254 & 1 & 3 \\
\hline Kúty & 48.659 & 17.021 & 1 & 3 \\
\hline Láb & 48.364 & 16.974 & 1 & 3 \\
\hline Malacky & 48.436 & 17.024 & 1 & 3 \\
\hline Matúškovo & 48.168 & 17.733 & 1 & 3 \\
\hline Miloslavov & 48.096 & 17.307 & 1 & 3 \\
\hline Mojzesovo & 48.134 & 18.239 & 1 & 3 \\
\hline Most pri Bratislave & 48.136 & 17.285 & 1 & 3 \\
\hline Močenok & 48.226 & 17.931 & 1 & 3 \\
\hline Nová Dedinka & 48.185 & 17.356 & 1 & 3 \\
\hline Nána & 47.815 & 18.695 & 1 & 3 \\
\hline Opoj & 48.303 & 17.643 & 1 & 3 \\
\hline Osuské & 48.623 & 17.451 & 1 & 3 \\
\hline Pribeta & 47.924 & 18.303 & 1 & 3 \\
\hline Prievidza & 48.772 & 18.626 & 1 & 3 \\
\hline Strekov & 47.895 & 18.394 & 1 & 3 \\
\hline Špačince & 48.441 & 17.612 & 1 & 3 \\
\hline Tehla & 48.186 & 18.389 & 1 & 3 \\
\hline Topol'čianky & 48.423 & 18.412 & 1 & 3 \\
\hline Tvrdošovce & 48.093 & 18.037 & 1 & 3 \\
\hline Valaliky & 48.641 & 21.301 & 1 & 3 \\
\hline Velčice & 48.415 & 18.305 & 1 & 3 \\
\hline Vel'ké Lovce & 48.061 & 18.338 & 1 & 3 \\
\hline Vel'ké Úl'any & 48.143 & 17.582 & 1 & 3 \\
\hline Vel'ký Krtíš & 48.214 & 19.350 & 1 & 3 \\
\hline Zvolen & 48.572 & 19.140 & 1 & 3 \\
\hline Žiar nad Hronom & 48.582 & 18.853 & 1 & 3 \\
\hline Žihárec & 48.073 & 17.882 & 1 & 3 \\
\hline Štúrovo & 47.804 & 18.698 & 2 & felt \\
\hline
\end{tabular}


Table 9. Continued from the previous page.

\begin{tabular}{|l|c|c|c|c|}
\hline Locality & $\begin{array}{c}\text { Lat. } \\
{\left[{ }^{\circ} \mathbf{N}\right]}\end{array}$ & $\begin{array}{c}\text { Lon. } \\
{\left[{ }^{\circ} \mathbf{E}\right]}\end{array}$ & $\begin{array}{c}\text { No. of } \\
\text { questionnaires }\end{array}$ & $\begin{array}{c}\text { I } \\
{\left[{ }^{\circ} \text { EMS-98 }\right]}\end{array}$ \\
\hline Medved'ov & 47.800 & 17.663 & 1 & felt \\
\hline Nováky & 48.725 & 18.544 & 1 & felt \\
\hline Nový Gúg & 47.969 & 18.108 & 1 & felt \\
\hline Okoč & 47.912 & 17.825 & 1 & felt \\
\hline Púchov & 49.124 & 18.326 & 1 & felt \\
\hline Studienka & 48.527 & 17.137 & 1 & felt \\
\hline Šaštín-Stráže & 48.643 & 17.147 & 1 & felt \\
\hline
\end{tabular}

\section{Conclusion and discussion}

The NNSS is operated by the ESI SAS, Bratislava. Data from all stations (except station HRB) are transferred in real-time to the data centre at Bratislava. Data processing and routine analysis are performed digitally by interactive seismological software Seismic Handler. Digital data are accessible both on-line and off-line in standard data format. So called Seismo Reports of seismic events recorded by NNSS are published on the web page of the ESI SAS http://www.seismology.sk/SeismoReports/reports.html.

In 2020 financial injection from the Slovak Academy of Sciences for the upgrade of the old instrumentation has been received. Thanks to this financal aid ESI SAS will be able to upgrade the equiment of seismic stations LANS and SRO next years.

Maintenance of seismic stations and routine daily interpretation of seismic data in 2020 were influenced by Covid-19 pandemics. On-site service of instrumentation was performed in emergency cases only. Photographical documentation of damages caused by April 23, 2020 earthquake in Vihorlat region (as recommended by EMS-98 manual; Grünthal, 1998) was not taken due to travelling restriction. Therefore, macroseicmic intensities for this earthquake were evaluated from the macroseismic questionnaires only.

Epicentres and local magnitudes were determined for 91 earthquakes originated on the territory of Slovakia in 2020. Weak seismic activity was recorded from several seismic source zones: Little Carpathians, Komárno, Vihorlat Mts., Vepor Mts., Záhorie region and Western Tatras. 
Acknowledgements. The authors have been supported by the Slovak Foundation Grant VEGA 2/0144/19, the Slovak Research and Development Agency Grant APVV-16-0146, the Visegrad Fund Grant No. 21930053 and the Program Mobility SAVAV ČR-21-02.

\section{References}

Csicsay K., Cipciar A., Fojtíková L., Kristeková M., Gális M., Srbecký M., Chovanová Z., Bystrický E., Kysel R., 2018: The National Network of Seismic Stations of Slovakia - Current state after 13 years in operation from the project of modernization and enhancement. Contrib. Geophys. Geod., 48, 4, 337-348, doi : 10.2478/congeo-2018 -0016 .

ESI SAS (Earth Science Institute of the Slovak Academy of Sciences), 2004: National Network of Seismic Stations of Slovakia. Deutsches GeoForschungsZentrum GFZ. Other/Seismic Network, doi: 10.14470/FX099882.

Fojtíková L., Kristeková M., Málek J., Sokos E., Csicsay K., Záhradník J., 2015: Quantifying capability of a local seismic network in terms of locations and focal mechanism solutions of weak earthquakes. J. Seismol., 20, 1, 93-106, doi : 10.1007/s10950-015 $-9512-1$.

GEOFON Data Centre, 1993: GEOFON Seismic Network. Deutsches GeoForschungsZentrum GFZ. Other/Seismic Network, doi : 10.14470/TR560404.

Grünthal G. (Ed.), 1998: European Macroseismic Scale 1998. Cahiers du Centre Européen de Géodynamique et de Séismologie, 15, Luxembourg, 101 p., online, accessed 28 October 2021, available from: http://media.gfz-potsdam.de/gfz/sec26/reso urces/documents/PDF/EMS-98_Original_englisch.pdf.

Hók J., Kysel R., Kováč M., Moczo P., Kristek J., Kristeková M., Šujan M., 2016: A seismic source zone model for the seismic hazard assessment of Slovakia. Geol. Carpath., 67, 3, 273-288, doi: 10.1515/geoca-2016-0018.

Institute of Geophysics, Academy of Sciences of the Czech Republic, 1973: Czech Regional Seismic Network. International Federation of Digital Seismograph Networks, Other/Seismic Network, doi : 10.7914/SN/CZ.

Institute of Physics of the Earth Masaryk University (Czech), 2014: IPE_EDU. International Federation of Digital Seismograph Networks, Other/Seismic Network, doi: $10.7914 /$ SN/D1.

Kövesligethy Radó Seismological Observatory (Geodetic and Geophysical Institute, Research Centre for Astronomy and Earth Sciences, Hungarian Academy of Sciences (MTA CSFK GGI KRSZO)), 1992: Hungarian National Seismological Network. Deutsches GeoForschungsZentrum GFZ. Other/Seismic Network, doi: 10.14470/UH 028726.

Liščák P., Petro L., Papčo J., Cipciar A., Csicsay K., Kristeková M., Stercz M., Pacajová K., Bednárik M., Briestenský M., Bella P., 2021: Partial Monitoring System Geological Factors, Subsystem 02: Tectonic and seismic activity of the territory, Geological work number 207, Report for 2020 (Čiastkový monitorovací systém - 
Geologické faktory, Podsystém 02: Tektonická a seizmická aktivita, číslo geologickej úlohy 207, správa za obdobie: rok 2020). State Geological Institute of Dionýz Štúr, in preparation (in Slovak).

Local Seismic Network of Eastern Slovakia. Faculty of Mathematics, Physics of the Earth and Informatics, Comenius University, http://www.fyzikazeme.sk/mainpage/ind ex_en.htm.

Pajdušák P., 1997: Historical seismic instruments at the stations Hurbanovo (HRB) and Skalnaté pleso (SPC) of Slovakia. Cahiers du Centre Européen de Géodynamique et de Séismologie, 13, 49-60.

Polish Seismological Network (PLSN), Institute of Geophysics Polish Academy of Sciences, https://www.igf.edu.pl/stacje-en.php.

Stammler K., 1993: Seismichandler-Programmable multichannel data handler for interactive and automatic processing of seismological analyses. Comput. Geosci., 19, 2, 135-140, doi : 10.1016/0098-3004(93)90110-Q.

Weber B., Becker J., Hanka W., Heinloo A., Hoffmann M., Kraft T., Pahlke D., Reinhardt J., Thoms H., 2007: SeisComp3 - automatic and interactive real time data processing. Geophysical Research Abstracts, EGU General Assembly, 9, 09129.

ZAMG - Zentralanstalt für Meterologie und Geodynamik, 1987: Austrian Seismic Network. International Federation of Digital Seismograph Networks, Other/Seismic Network, doi : 10.7914/SN/OE. 